\title{
The elastic modulus of isolated polytetrafluoroethylene filaments
}

\author{
Patrick Drawe, Nils O.B. Lüttschwager, and Martin A. Suhm* \\ Institut für Physikalische Chemie, Universität Göttingen, Tammannstr. 6, 37077 Göttingen, Germany \\ *Corresponding author's e-mail address: msuhm@gwdg.de
}

Published online: September 15, 2014 (version 2)

Cite as: Drawe et al., ScienceOpen Research 2014 (DOI: 10.14293/S2199-1006.1.SOR-MATSCI.AKA0J6.v2)

Reviewing status: Please note that this article is under continuous review. For the current reviewing status and the latest referee's comments please click here or scan the QR code at the end of this article.

Primary discipline: Materials Science

Associated discipline: Polymer Science, Materials Properties, Materials Characterization, Chemistry, Physics

Keywords: Raman spectroscopy, Polymer, Teflon, PTFE, Elastic modulus, Mechanical property, Nanorod, Perfluoroalkane

\begin{abstract}
We report vibrational Raman spectra of small extended perfluoro- $n$-alkanes $\left(\mathrm{C}_{n} \mathrm{~F}_{2 n+2}\right.$ with $\left.n=6,8-10,12-14\right)$ isolated in supersonic jet expansions and use wavenumbers of longitudinal acoustic vibrations to extrapolate the elastic modulus of cold, isolated polytetrafluoroethylene filaments. The derived value $E=209(10) \mathrm{GPa}$ defines an upper limit for the elastic modulus of the perfectly crystalline, noninteracting polymer at low temperatures and serves as a benchmark for quantum chemical predictions.
\end{abstract}

\section{INTRODUCTION}

Polytetrafluoroethylene (PTFE), commonly known as Teflon ${ }^{\circledR}$, is valued for its low coefficient of friction and high inertness. It is used in various technical applications like nonstick coatings. Its elastic modulus describes how the material stretches under stress and is an important characteristic property for engineering purposes. It varies over several orders of magnitude depending on the crystallinity of the material. Characterization of the maximum elastic modulus of PTFE achievable when the stress acts along the axis of parallel PTFE strands - has received repeated attention over the last decades [1-7]. It was recently estimated to be $E_{\max } \approx$ $220 \mathrm{GPa}[5,7]$, two orders of magnitude higher than in amorphous PTFE. In this work, we extend previous efforts to determine the highest achievable elastic modulus of polyethylene $[8,9]$ to PTFE by investigating longitudinal acoustic modes (LAMs) of isolated perfluoro- $n$-alkanes which are rectified by collisional cooling in supersonic jet expansions. This technique allows us to determine the elastic modulus for cold molecules free of interactions with a molecular environment and free of any sample variations characteristic for macroscopic samples. The result is well-suited as a benchmark for quantum chemical predictions and sets an upper bound for material studies.

Crystalline PTFE assumes a helical structure [10] which is also confirmed for small perfluoro- $n$-alkanes in the gas phase
[11]. In our jet experiment, we find that the chain molecules exclusively occupy a helical all-anti conformation (vide infra), which is shown in Figure 1 for perfluorotetradecane $(n=14)$. The LAMs of such fully extended chain molecules have a macroscopic equivalent. For a macroscopic rod of length $l$, made from a homogeneous material with the elastic modulus $E$, and density $\rho$, one finds the following formula for its LAM wavenumbers [12]:

$$
\tilde{\nu}_{\mathrm{LAM}-m}=\frac{m}{2 c_{0} l} \sqrt{\frac{E}{\rho}} .
$$

Here, $m$ is the number of nodes of the longitudinal vibration and $c_{0}$ is the speed of light in vacuum. The vibrational displacement of the LAM-1 is shown in Figure 1 for $n=14$. In this work, we present and interpret LAM-1 wavenumbers up to $n=14$.

\section{METHODS AND RESULTS}

Supersonic expansions of perfluoro- $n$-alkanes diluted in helium were generated by expanding the gas mixtures from 0.8 bar through a $4 \times 0.15 \mathrm{~mm}^{2}$ slit nozzle into a vacuum chamber kept at 2 mbar. Gas mixtures were prepared by premixing perfluoro- $n$-alkanes with helium in a vacuum line $(n=$ $6-9$ ) or by bubbling helium through a coolable glass saturator $\left(n=10-13\right.$, temperature $\vartheta=-7$ to $\left.25^{\circ} \mathrm{C}\right)$ or through a heatable brass saturator $\left(n=14, \vartheta=55^{\circ} \mathrm{C}\right)$. The realized mole

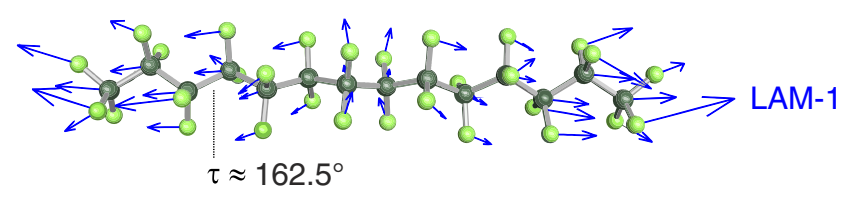

Figure 1. Minimum energy structure of $\mathrm{C}_{14} \mathrm{~F}_{30}$ on the B3LYP-D3/ def2-TZVP level. All CC torsion angles $(\tau)$ are close to $163^{\circ}$, similar to the $13 / 6$ helix in the solid [10]. Blue arrows indicate the calculated LAM-1 normal coordinate. 


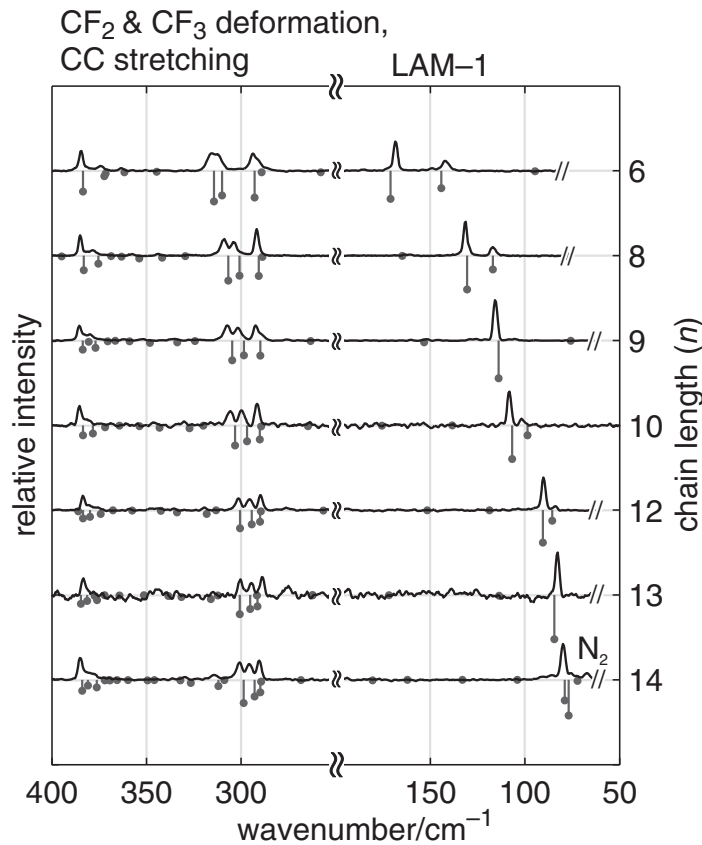

Figure 2. Raman jet spectra of perfluorinated alkanes expanded in helium. Calculated harmonic wavenumbers and scattering cross-sections (all-anti, B3LYP-D3/def2-TZVP, unscaled, $T=100 \mathrm{~K}$ ) are given as gray inverted stems.

fractions are in the $0.1 \%$ range, but vary somewhat for different chain lengths. This is reflected in a varying signal-to-noise ratio. A $532 \mathrm{~nm} 18 \mathrm{~W}$ continuous-wave laser was focused on the expansion $1 \mathrm{~mm}$ downstream the nozzle, perpendicular to the expansion. The risk of sample decomposition by the laser is negligible due to the sub-microsecond residence time and lack of absorption bands in the visible. Raman scattered light was collected at a $90^{\circ}$ observation angle. Emission lines from a neon lamp were recorded for wavelength calibration. More details on the Raman jet setup and LAM spectroscopy can be found elsewhere [9]. The resulting Savitzky-Golay filtered (second-order polynomial, 7 pixel) Raman jet spectra are shown in Figure 2 together with harmonic B3LYP-D3/def2TZVP predictions which were calculated with Turbomole v6.4 [13].

Other than in case of regular $n$-alkanes [9], conformations with kinks relative to the most stable all-anti conformation do not appear in the spectra. This observation is in line with experimental gauche conformation (CC torsion) energy penalties which are doubled in case of perfluorinated alkanes $(\approx 5$ $\mathrm{kJ} / \mathrm{mol}[14,15]$ for perfluoro- $n$-alkanes, $\approx 2.5 \mathrm{~kJ} / \mathrm{mol}[16,17]$ for $n$-alkanes), and with calculated barrier heights, which are smaller for the fluorinated compounds $(\approx 8 \mathrm{~kJ} / \mathrm{mol}[18,19]$ for perfluoro- $n$-alkanes, $\approx 12 \mathrm{~kJ} / \mathrm{mol}[20,21]$ for $n$-alkanes), both leading to an enhancement of the ground state population in a jet experiment. Therefore, jet cooling is a perfect alternative to crystal packing to straighten perfluoro- $n$ alkanes.
Table 1. Deperturbed and recalibrated LAM-1 wavenumbers of perfluoro- $n$-alkanes, see text. Wavenumbers and recalibration are given in $\mathrm{cm}^{-1}$.

\begin{tabular}{lccccccc}
\hline Chain length $\boldsymbol{n}$ & $\mathbf{6}$ & $\mathbf{8}$ & $\mathbf{9}$ & $\mathbf{1 0}$ & $\mathbf{1 2}$ & $\mathbf{1 3}$ & $\mathbf{1 4}$ \\
\hline Original $\tilde{\nu}_{\text {LAM }}$ & 168.3 & 131.4 & 115.7 & 108.3 & 90.2 & 82.9 & 79.8 \\
Original $\tilde{\nu}_{\text {TAM }}$ & 141.9 & 116.8 & - & 101.5 & 84.0 & - & - \\
$I_{\text {TAM }} I_{\text {LAM }}$ & 0.748 & 0.297 & - & 0.257 & 0.215 & - & - \\
Recalibration & +0.1 & -0.3 & -0.9 & -0.8 & +1.0 & +1.2 & -0.4 \\
Final $\tilde{\nu}_{\text {LAM }}^{(0)}$ & 157.1 & 127.7 & 114.8 & 106.1 & 90.5 & 84.1 & 79.4 \\
\hline
\end{tabular}

The observed spectral window reveals the required LAMs with one node between 50 and $200 \mathrm{~cm}^{-1}$. Different kinds of $\mathrm{CF}_{2}$ and $\mathrm{CF}_{3}$ deformation vibrations, partly coupled to $\mathrm{C}-\mathrm{C}$ stretching vibrations, contribute in the $250-400 \mathrm{~cm}^{-1}$ range and are shown merely for completeness. They will not be analyzed, but the average position of the $\mathrm{CF}_{2}$ scissoring vibration $\left(\approx 385 \mathrm{~cm}^{-1}\right)$ is used as a wavenumber standard to refine the calibration of the spectra because the wavenumber of this vibration is largely chain-length-independent (in the B3LYP-D3/def2-TZVP calculations, it scatters around the average value $383.6 \mathrm{~cm}^{-1}$ with a maximum deviation of less than $1 \mathrm{~cm}^{-1}$ for $n=6-14$ ). LAMs with a higher number of nodes are not observed. Notably, some of the LAM-1 bands are split due to harmonic mode mixing with a transverse acoustic mode (TAM). In these cases, the wavenumber of the pure LAM is recovered by determining the intensity-weighted center of the LAM-1 and TAM Raman bands [8] under the assumption that the pure TAM does not carry intensity $(I)$ on its own:

$$
\tilde{\nu}_{\mathrm{LAM}}^{0}=\frac{\tilde{\nu}_{\mathrm{LAM}} I_{\mathrm{LAM}}+\tilde{\nu}_{\mathrm{TAM}} I_{\mathrm{TAM}}}{I_{\mathrm{LAM}}+I_{\mathrm{TAM}}}
$$

Original and final LAM-1 wavenumbers are summarized in Table 1. The low scattering of the data when plotted appropriately (vide infra) underlines the recalibration and deperturbation success. Residual thermal shifts of the band positions compared to the $0 \mathrm{~K}$ value are expected to be of a similar size as calibration shifts, at most. Like in the case of alkanes $[9,12]$, condensed phase frequencies of the perfluoroalkane LAM modes [4] are typically a few percent higher than the present unperturbed gas phase values due to lamellar and other intermolecular effects.

\section{DISCUSSION}

Assuming that the chain molecules behave like small homogeneous rods, their LAM-1 wavenumbers can be connected to the elastic modulus $E$ of the regular bulk solid via [8, 12]:

$$
\tilde{\nu}_{\mathrm{LAM}}=\frac{1}{2 c_{0} n d} \sqrt{\frac{E}{\rho}}=\frac{A}{n},
$$

where we use equation (1) and replace the length of the rod $l$ for convenience with the chain length $n$ using the longitudinal 


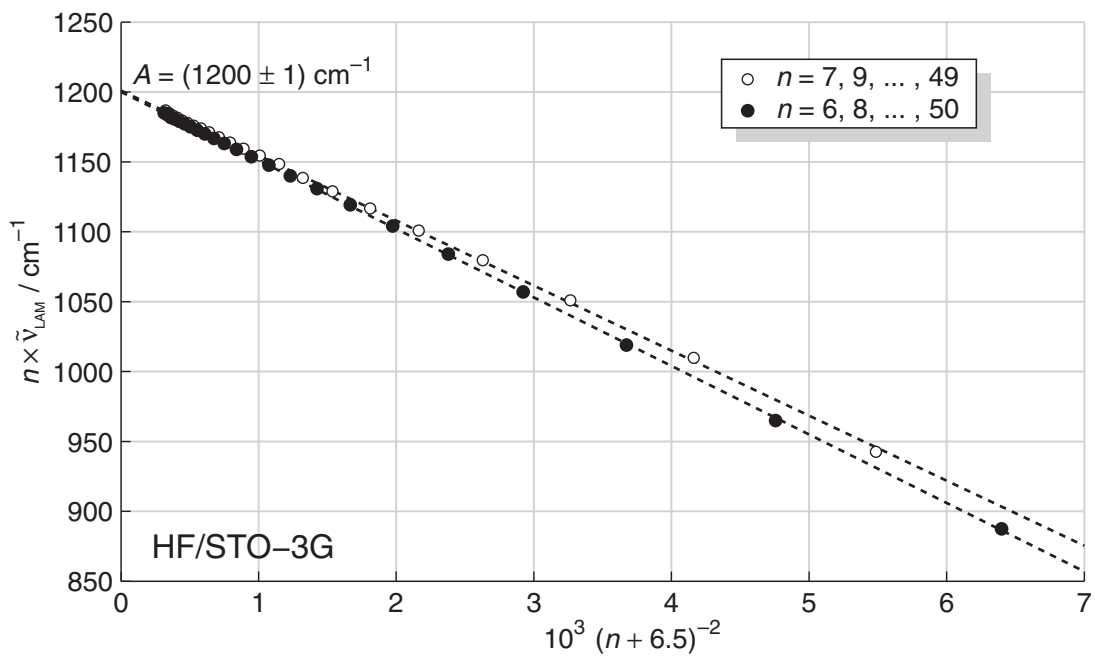

Figure 3. Extrapolation of deperturbed harmonic HF/STO-3G LAM-1 wavenumbers (Gaussian 03 [23]) to infinite chain length ( $n$ ).

segment separation $d=0.130 \mathrm{~nm}[10]$ between two $-\mathrm{CF}_{2-}$ units $(l=n d)$. In case of long chain molecules, their longitudinal atomic structure is negligible (the homogeneous rod approximation holds) and a plot of LAM-1 wavenumbers versus the inverse chain length yields a straight line [9] whose slope $A$ can be translated to the elastic modulus:

$$
E=4 \rho\left(c_{0} d A\right)^{2} .
$$

LAM-1 wavenumbers of the rather short perfluoroalkanes considered here clearly deviate from linearity when plotted against the inverse chain length due to end-group effects and the curvature of the corresponding frequency branch [22] to which the LAMs belong. We take these deviations into account by slightly rearranging equation (3) and adding an additional term which vanishes for long molecules [8]:

$$
n \tilde{\nu}_{\mathrm{LAM}}=A+\frac{B}{(n+C)^{2}} .
$$

Now, the parameter $A$ is the value for $n \tilde{\nu}_{\text {LAM }}$ in the limit of long chain length. The additional parameters $B$ and $C$ are not given a physical interpretation. The parameters can be derived from a curve fit, but allowing $B$ and $C$ to vary freely raises the uncertainty of $A$. It is helpful (but not critical) to plug in a quantum chemical estimate for $C$. This is done by plotting a large set of harmonic HF/STO-3G LAM-1 wavenumbers $(n=6-50$, calculated with Gaussian 03 [23]) against $(n+$ $C)^{-2}$ after deperturbation according to equation (2). For this simulated dataset, $C$ is varied until the quantum chemical predictions fall on a straight line, which is the case for $C=6.5$ (Figure 3). The restriction to a low computational level is necessary to reach the homogeneous rod limit (high $n$ ), which is approached rather late. This is obvious from the alternation for even and odd chain length in case of short molecules, which makes it necessary to split the extrapolation. The validity of this extrapolation approach is strengthened by our studies on $n$-alkanes where the purely experimental extrapolation of long alkane ( $n=16-21)$ LAM-1 wavenumbers with equation (3) $[9,24]$ yields virtually the same elastic modulus as the extrapolation of short alkane $(n=5-16)$ LAM1 wavenumbers employing low-level quantum chemical predictions and equation (5) [8], 305(5) GPa and 309(8) GPa, respectively.

The experimental extrapolation for perfluoro- $n$-alkanes with even numbered chain length is shown in Figure 4 (the dataset for odd $n$ being too small) using $C=6.5 \pm 1.0$ and $\tilde{\nu}_{\text {LAM }} \pm$ $1 \mathrm{~cm}^{-1}$ to establish lower and upper bounds for the $y$-intercept $A$, yielding $A=1.21(3) \times 10^{3} \mathrm{~cm}^{-1}$. With equation (4), the PTFE crystal density $\rho=2.344 \mathrm{~g} / \mathrm{cm}^{3}$ [10], and the segment separation $d=0.130 \mathrm{~nm}$ [10], this translates to the elastic modulus $E=209(10) \mathrm{GPa}$.

This Raman jet estimate compares favorably with moduli reported in the literature (Table 2), but the comparison is somewhat hampered by the fact that the literature moduli are reported in part for PTFE in slightly different modifications, and/or use different parameters (density or molecular chain cross-section) to convert molecular properties to the macroscopic modulus. Some literature moduli were derived using densities (or related chain cross-sections) which differ by as much as $10 \%$ from the value used here. Our value for $E$ should thus be compared within a larger error margin, including this uncertainty in the density: $E \approx 2.1(2) \times 10^{2} \mathrm{GPa}$. This value agrees with most of the values given in Table 2 , only the early force field calculation [1] and a stress-strain measurement [3], which might have suffered from a nonuniform distribution of stress over the sample [25], deviate notably.

It is somewhat unsatisfactory to increase the error bars for the more precise spectroscopic data we present here. A way 


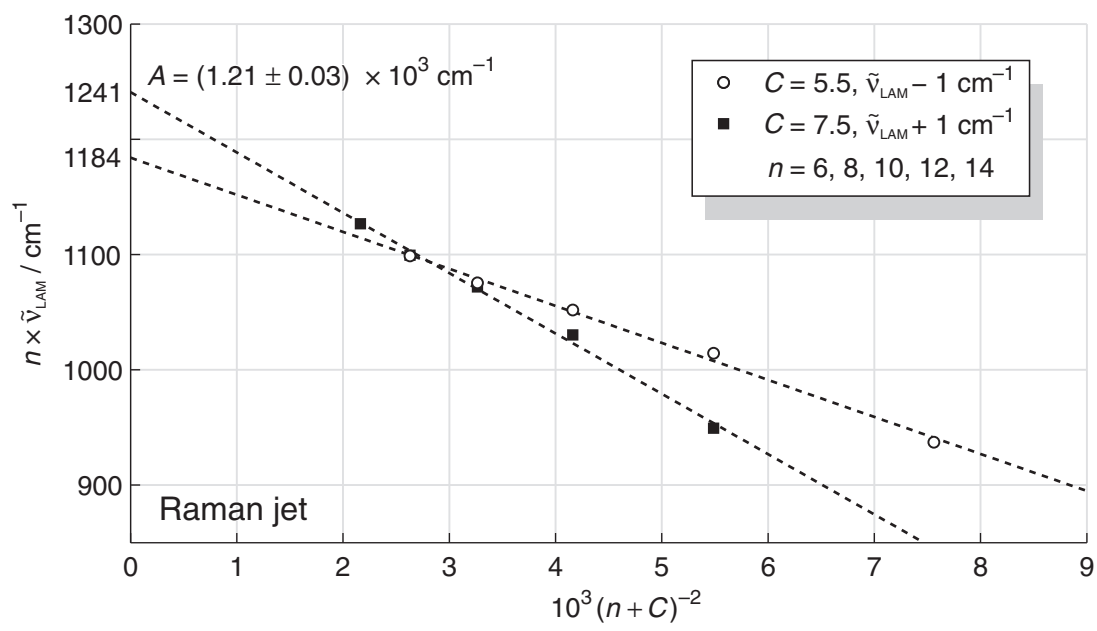

Figure 4. Extrapolation of deperturbed Raman jet LAM-1 wavenumbers to infinite chain length ( $n$ ).

Table 2. Elastic modulus of crystalline PTFE along the chain direction according to different methods.

\begin{tabular}{|c|c|c|c|c|c|c|c|c|}
\hline \multirow[b]{2}{*}{ Reference } & \multicolumn{3}{|c|}{ Computational } & \multicolumn{5}{|c|}{ Experimental } \\
\hline & [1] & [5] & [6] & [3] & [7] & {$[2,4]$} & [4] & this work \\
\hline$E / \mathrm{GPa}$ & 160 & 221 & $247^{c}$ & 153 & 220 & 222 & 206 & 209(10) \\
\hline Method $^{a}$ & $\mathrm{FF}$ & DFT & DFT & $S S^{b}$ & $S S^{b}$ & $\operatorname{LAM}^{\mathrm{b}}$ & LAM $^{b}$ & LAM \\
\hline
\end{tabular}

${ }^{\mathrm{a}} \mathrm{FF}=$ force field calculation, DFT $=$ density functional theory calculation, $\mathrm{SS}=$ stress-strain measurement, LAM = LAM frequency extrapolation; ${ }^{b}$ solid state; planar backbone.

to circumvent the poorly defined density of a single molecular filament (which, in this case, is due to the poorly defined cross-section area $S$ of a molecule) is to translate the spectroscopic value $A$ to the force $F$ which is necessary to stretch a single PTFE rod up to a strain $\Delta l / l=1 \%$. To do so, we rearrange the definition of the elastic modulus $E=\frac{F / S}{\Delta l / l}$ and use equations (3) and (4), and $\rho=\frac{m_{\mathrm{CF}_{2}}}{d S}$ to arrive at:

$$
F=(\Delta l / l) 4 m_{\mathrm{CF}_{2}} d\left(c_{0} A\right)^{2} .
$$

The cross-section area $S$ cancels, and we are left with the well-defined mass of a ${ }^{12} \mathrm{C}^{19} \mathrm{~F}_{2}$ unit $m_{\mathrm{CF}_{2}}=8.302 \times 10^{-26} \mathrm{~kg}$ [26]. Then, the spectroscopic value $A$ translates to $F=5.7$ (3) $\times 10^{2} \mathrm{pN}$, which reproduces the value we reported [9] for single polyethylene strands $F=5.6(1) \times 10^{2} \mathrm{pN}$ within the error bars. It should be emphasized that polyethylene prefers a nonhelical all-trans conformation [27]. Apparently, the small twisting of the carbon chain and substitution of hydrogen by fluorine in PTFE does not influence the stiffness of a single chain significantly [3], whereas they contribute to the lower friction by allowing for motion far below the melting transition [28]. This motion is probably facilitated by the propensity for stretched conformations in perfluoroalkanes (see
Figure 2) in contrast to the ease with which normal alkanes kink and form gauche conformations [9] and also by the absence of the regular zig-zag pattern of stretched alkyl chain segments, which tends to lock in when chains glide past each other. The difference in the limiting elastic moduli of PTFE and polyethylene emerges mainly from closer chain packing in case of polyethylene. Other than in case of $n$-alkanes $[8,9]$, the modulus derived from condensed phase perfluoroalkane data, extrapolating LAM wavenumbers from solid samples [4], agrees well with our vacuum isolation estimate without corrections for intermolecular interactions [29]. This suggests that such interactions are weaker if hydrogen is swapped with fluorine [30] and is fully in line with the high volatility of perfluoro- $n$-alkanes.

\section{CONCLUSIONS}

The longitudinal stiffness of a polymer chain is an important mechanical property which is usually hidden in a macroscopic polymer sample due to conformational flexibility and a lack of regularity. Its extraction from Raman spectra of finite oligomers in the condensed phase may require elaborate corrections for intermolecular interactions [29]. We provide the first interaction-free spectroscopic determination for PTFE from monoconformational, collisionally cooled perfluoro- $n$ alkanes in supersonic jets. The resulting elastic modulus amounts to two-thirds of the corresponding polyethylene value $[8,9]$, which is mainly due to the larger cross section of a single PTFE strand caused by the fluorine atoms. Because the elastic modulus refers to the force per area, more polyethylene than PTFE strands must be stretched per unit area. This results in a higher stiffness of polyethylene for geometrical rather than energetical reasons.

Single molecular filament characterization in terms of their longitudinal elasticity is now feasible by supersonic jet spectroscopy for fragments with a vapor pressure on the order of 
at least $10 \mu \mathrm{bar}$, if the necessary heating prior to the expansion is tolerated for some hours without decomposition, if the longitudinal acoustic vibration is sufficiently Raman active, and if the conformational diversity can be controlled by rapid cooling. To make the result useful for the bulk material, it requires furthermore sufficiently weak inter-strand interactions. This adds a gas phase perspective to polymer science which can be exploited for rigorous comparison between theory and experiment.

\section{ACKNOWLEDGMENTS}

Support from the German Research Foundation (DFG, Grant $\mathrm{Su} 121 / 2$ and GRK 782) is gratefully acknowledged. We further acknowledge publication support by the German Research Foundation and the Open Access Publication Funds of the Göttingen University.

\section{REFERENCES}

[1] Shimanouchi $T$, Asahina $M$, Enomoto S. Elastic moduli of oriented polymers. I. The simple helix, polyethylene, polytetrafluoroethylene, and a general formula. J Polym Sci. 1962;59:93100. doi:10.1002/pol.1962.1205916708

[2] LaGarde V, Prask H, Trevino S. Vibrations in teflon. Discuss Faraday Soc. 1969;48:15-18. doi:10.1039/df9694800015

[3] Sakurada I, Kaji K. Relation between the polymer conformation and the elastic modulus of the crystalline region of polymer. J Polym Sci Part C Polym Symp. 1970;31:57-76. doi:10.1002/ polc.5070310108

[4] Rabolt JF, Fanconi B. Longitudinal acoustic modes of polytetrafluoroethylene copolymers and oligomers. Polymer. 1977;18:1258-1264. doi:10.1016/0032-3861(77)90290-7

[5] Bartha F, Bogár F, Peeters A, Van Alsenoy C, Van Doren V. Density-functional calculations of the elastic properties of some polymer chains. Phys Rev B Condens Matter Mater Phys. 2000;62:10142-10150. doi:10.1103/PhysRevB.62.10142

[6] Zhang M-L, Miao MS, Peeters A, Van Alsenoy C, Ladik JJ, Van Doren VE. LDA calculations of the Young's moduli of polyethylene and six polyfluoroethylenes. Solid State Commun. 2000;116:339-343. doi:10.1016/S0038-1098(00)00313-6

[7] Brown EN, Rae PJ, Dattelbaum DM, Clausen B, Brown DW. Insitu measurement of crystalline lattice strains in polytetrafluoroethylene. Exp Mech. 2008;48:119-131. doi:10.1007/s11340007-9075-3

[8] Wassermann TN, Thelemann J, Zielke P, Suhm MA. The stiffness of a fully stretched polyethylene chain: a Raman jet spectroscopy extrapolation. J Chem Phys. 2009;131:161108. doi:10.1063/1.3256221

[9] Lüttschwager NOB, Suhm MA. Stretching and folding of 2-nanometer hydrocarbon rods. Soft Matter. 2014;10:4885-4901. doi:10.1039/C4SM00508B

[10] Clark ES. The molecular conformations of polytetrafluoroethylene: forms II and IV. Polymer. 1999;40:4659-4665. doi:10.1016/S0032-3861(99)00109-3

[11] Fournier JA, Phan CL, Bohn RK. Microwave spectroscopy and characterization of the helical conformer of perfluorohexane. ARKIVOC 2011;Part (v):5-11.

[12] Schaufele RF, Shimanouchi T. Longitudinal acoustical vibrations of finite polymethylene chains. J Chem Phys. 1967;47:36053610. doi:10.1063/1.1712428
[13] TURBOMOLE v6.4 2012, a development of University of Karlsruhe and Forschungszentrum Karlsruhe GmbH, 19892007, TURBOMOLE GmbH, since 2007; Available from http:// www.turbomole.com.

[14] Campos-Vallette M, Rey-Lafon M. Vibrational spectra and rotational isomerism in short chain n-perfluoroalkanes. J Mol Struct. 1983;101:23-45. doi:10.1016/0022-2860(83)85041-8

[15] Hsu SL, Reynolds N, Bohan SP, Strauss HL, Snyder RG. Structure, crystallization, and infrared spectra of amorphous perfluoro$\mathrm{n}$-alkane films prepared by vapor condensation. Macromolecules. 1990;23:4565-4575. doi:10.1021/ma00223a012

[16] Herrebout WA, van der Veken BJ, Wang A, Durig JR. Enthalpy difference between conformers of $n$-butane and the potential function governing conformational interchange. J Phys Chem. 1995;99:578-585. doi:10.1021/j100002a020

[17] Balabin RM. Enthalpy difference between conformations of normal alkanes: Raman spectroscopy study of $n$-pentane and $n$ butane. J Phys Chem A. 2009;113:1012-1019. doi:10.1021/ jp809639s

[18] Röthlisberger U, Laasonen K, Klein ML, Sprik M. The torsional potential of perfluoro $n$-alkanes: a density functional study. J Chem Phys. 1996;104:3692-3700. doi:10.1063/1.471023

[19] Borodin O, Smith GD, Bedrov D. A quantum chemistry based force field for perfluoroalkanes and poly(tetrafluoroethylene) J Phys Chem B. 2002;106:9912-9922. doi:10.1021/jp026158i

[20] Allinger NL, Fermann JT, Allen WD, Schaefer III HF. The torsional conformations of butane: Definitive energetics from $a b$ initio methods. J Chem Phys. 1997;106:5143-5150. doi:10.1063/1.473993

[21] Martin JML. What can we learn about dispersion from the conformer surface of $n$-pentane? J Phys Chem A. 2013;117:31183132. doi:10.1021/jp401429u

[22] Hannon MJ, Boerio FJ, Koenig JL. Vibrational analysis of polytetrafluoroethylene. J Chem Phys. 1969;50:2829-2836. doi:10.1063/1.1671471

[23] Frisch MJ, Trucks GW, Schlegel HB, Scuseria GE, Robb MA, Cheeseman JR, Montgomery Jr, JA, Vreven T, Kudin KN, Burant JC, et al. Gaussian 03, Revision B.04. Wallingford, CT: Gaussian, Inc.; 2004.

[24] Lüttschwager NOB. Raman spectroscopy of conformational rearrangements at low temperatures - folding and stretching of alkanes in supersonic jets. Heidelberg: Springer; 2014. doi:10.1007/978-3-319-08566-1

[25] Fanconi B, Rabolt JF. The determination of longitudinal crystal moduli in polymers by spectroscopic methods. J Polym Sci Polym Phys Ed. 1985;23:1201-1215. doi:10.1002/pol.1985. 180230612

[26] Lide DR (ed.). CRC handbook of chemistry and physics, 82nd ed. Boca Raton: CRC Press; 2001.

[27] Bunn CW. The crystal structure of long-chain normal paraffin hydrocarbons. The "shape" of the $<\mathrm{CH} 2$ group. Trans Faraday Soc. 1939;35:482-491. doi:10.1039/tf9393500482

[28] Bunn CW, Howells ER. Structures of molecules and crystals of fluoro-carbons. Nature. 1954;174:549-551. doi:10.1038/174 $549 \mathrm{a} 0$

[29] Snyder RG, Strauss HL, Alamo R, Mandelkern L. Chain-length dependence of interlayer interaction in crystalline $n$-alkanes from Raman longitudinal acoustic mode measurements. J Chem Phys. 1994;100:5422-5431. doi:10.1063/1.467159

[30] Cametti M, Crousse B, Metrangolo P, Milani R, Resnati G. The fluorous effect in biomolecular applications. Chem Soc Rev. 2012;41:31-42. doi:10.1039/c1cs15084g5 


\section{COMPETING INTERESTS}

The authors declare no competing interests.

\section{PUBLISHING NOTES}

(C) 2014 Drawe et al. This work has been published open access under Creative Commons Attribution License CC BY 4.0, which permits unrestricted use, distribution, and reproduction in any medium, provided the original work is properly cited. Conditions, terms of use and publishing policy can be found at www.scienceopen.com.
Please note that this article may not have been peer reviewed yet and is under continuous post-publication peer review. For the current reviewing status please click here or scan the QR code on the right.

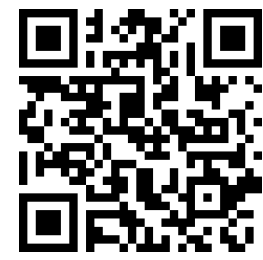

